OPEN

SUBJECT AREAS:

MESENCHYMAL STEM

CELLS

STEM-CELL RESEARCH

BIOMATERIALS - CELLS

BIOMEDICAL MATERIALS

Received

1 March 2013

Accepted

27 June 2013

Published

22 July 2013

Correspondence and requests for materials should be addressed to

Y.L. (alun@hum-gen. au.dk) or N.L.

(lunonghua@163.

com)

* These authors contributed equally to this work.

\section{A simple method for deriving functional MSCs and applied for osteogenesis in 3D scaffolds}

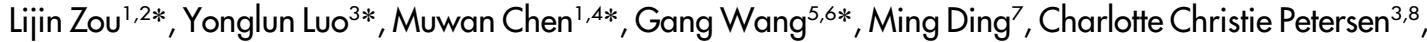 \\ Ran Kang' ${ }^{1}$ Frederik Dagnaes-Hansen ${ }^{3}$, Yuanlin Zeng ${ }^{2}$, Nonghua Lv², Qing Ma ${ }^{5,6}$, Dang Q. S. Le', \\ Flemming Besenbacher ${ }^{4}$, Lars Bolund ${ }^{3}$, Thomas G. Jensen ${ }^{3}$, Jørgen Kjems ${ }^{4}$, William T. Pu ${ }^{5,6}$ \& Cody Bünger ${ }^{1}$
}

'Orthopaedic Research Laboratory, Department of Orthopaedics, Aarhus University Hospital, Aarhus, DK, ${ }^{2}$ Department of Burn surgery and Gastroenterology, The First Affiliated Hospital of Nanchang University, Nanchang, CN, ${ }^{3}$ Department of Biomedicine, Aarhus University, 8000, Aarhus, DK, ${ }^{4}$ Interdisciplinary Nanoscience Center, Aarhus University, Aarhus, DK, ${ }^{5}$ Department of Cardiology, Boston Children's Hospital, MA, USA, ${ }^{6}$ Harvard Stem Cell Institute, Harvard University, Cambridge, MA, USA, ${ }^{7}$ Orthopaedic Research Laboratory, Department of Orthopaedics \& Traumatology, Odense University Hospital, Institute of Clinical Research, University of Southern Denmark, 5000 Odense C, Denmark, ${ }^{8}$ The FACS Core Facility, Faculty of Health, Aarhus University, Aarhus, Denmark.

We describe a simple method for bone engineering using biodegradable scaffolds with mesenchymal stem cells derived from human induced-pluripotent stem cells (hiPS-MSCs). The hiPS-MSCs expressed mesenchymal markers (CD90, CD73, and CD105), possessed multipotency characterized by tri-lineages differentiation: osteogenic, adipogenic, and chondrogenic, and lost pluripotency - as seen with the loss of markers OCT3/4 and TRA-1-81 - and tumorigenicity. However, these iPS-MSCs are still positive for marker NANOG. We further explored the osteogenic potential of the hiPS-MSCs in synthetic polymer polycaprolactone (PCL) scaffolds or PCL scaffolds functionalized with natural polymer hyaluronan and ceramic TCP (PHT) both in vitro and in vivo. Our results showed that these iPS-MSCs are functionally compatible with the two 3D scaffolds tested and formed typically calcified structure in the scaffolds. Overall, our results suggest the iPS-MSCs derived by this simple method retain fully osteogenic function and provide a new solution towards personalized orthopedic therapy in the future.

M esenchymal stem cells (MSCs), which possess multilineage potential of differentiating to mesenchymal tissues such as bone, fat, and cartilage, represent an important renewable cell source for orthopedic therapy ${ }^{1}$. However, the clinical applications of autologous transplantation of MSCs derived from adult tissues, such as bone marrow and adipose tissue, are hampered by their limited proliferating capacity, which decreases with increasing donor age ${ }^{2}$.

Induced pluripotent stem (iPS) cells, generated by ectopically expressing certain transcription factors (typically Oct3/4, Sox2, Klf4 and c-Myc ${ }^{3}$ ), resemble ES cells in a pluripotent state ${ }^{4}$. Methods for reprogramming human somatic cells into iPS cells have dramatically improved over the last five years ${ }^{5}$. Non-integrating methods by e.g. direct mRNAs or proteins transfection, and integration deficient virus are favored for clinically compliant applications ${ }^{6-9}$. Mesenchymal stem cells derived from mouse or human iPS cells (iPS-MSCs) have been reported by several groups ${ }^{10,11}$ and used for osteogenesis both in vitro and in vivo ${ }^{12}$, which makes iPS-MSCs a promising cell source for studying orthopedic diseases, regenerative orthopedic therapy, and patient-specific cell therapy. Moreover, the iPS-MSCs have greater capacity of cell proliferation than bone marrow-derived MSCs (over 10fold higher in telomerase activity) and are able to proliferate for 120 population doublings without losing selfrenewal capacity and MSC characteristics ${ }^{13}$.

Most MSCs derivations are achieved via the formation of three-dimensional embryoid bodies, which are laborious, inefficient and uncontrollable for spontaneous differentiation ${ }^{14}$. Yen et al. have described a simple method of differentiating human ES cells (hESCs) into MSCs by switching hES cells from ES medium to defined medium, which consisted of DMEM-low glucose, $10 \%$ FBS, and $1 \%$ Penicillin/Streptomycin, followed by trypsinization-based passaging ${ }^{15}$. In this study, we investigated whether this method can be applied to human iPS cells. We also investigated the potential for combining iPS-MSCs and 3D orthopedic scaffolds made from biodegradable polymer materials for bone engineering. 


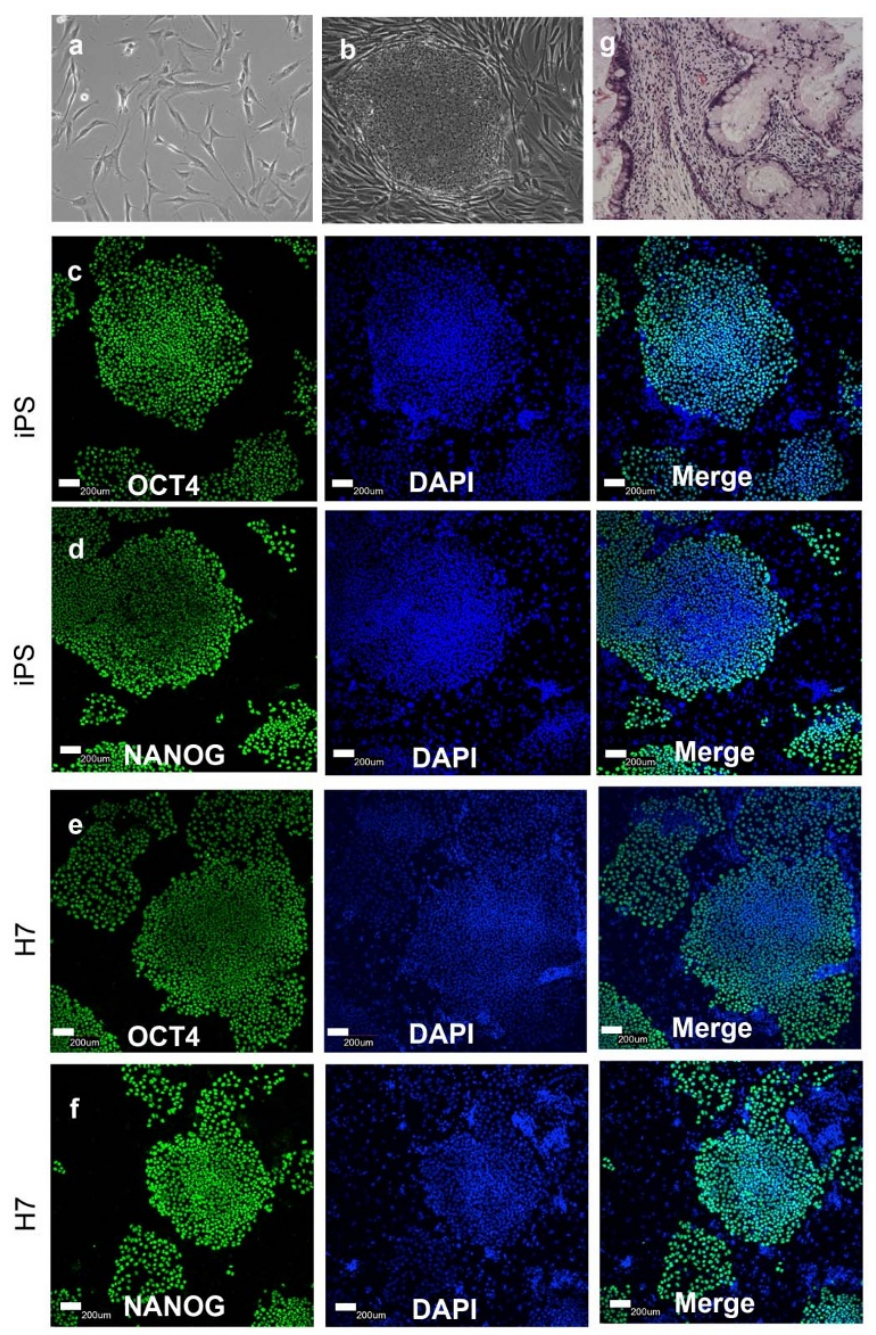

Figure 1 | Generation of human iPSCs by mRNA reprogramming. Phase-contrast image of human fibroblasts (a) and mature iPSC clone (b). (c-d) Immunohistochemistry of pluripotency markers: OCT4 (c) and NANOG (d) in iPSCs. (e-f) Immunohistochemistry of pluripotency markers: OCT4 (e) and NANOG (f) in H7 lines. (g) Teratoma formed in SCID mice 8 weeks after injected with iPS cells.

\section{Results}

Generation of human iPSCs (hiPSCs) by mRNA reprogramming. We firstly generated human iPSCs by mRNA reprogramming, which is nonintegrating and clinically relevant. We transfected human IMR90 fibroblasts (Fig. 1a) with modified mRNA cocktails. Expression of Oct4, Sox2, and Klf4 were observed in transfected cells 16 hours after transfection (Supplementary Fig. 1). Colonies of human ES cell morphology (iPSC clones) first became visible 12 days after transfection with a modified mRNA reprogramming cocktail and on day 20 roughly 100 iPSC clones were observed. Clones with typical morphology of human ES cells were selected for expansion (Fig. 1b). The iPS cells were positive for pluripotent marker OCT4 (Fig. 1c) and NANOG (Fig. 1d), in parity with the H7 ES cell line (Fig. le and 1f), and were able to form teratoma in vivo when injected into the immunosuppressed mouse (Fig. 1g).

One-step derivation of fibroblastic-like cells from hiPSCs. To differentiate the iPSCs to MSCs, we switched the iPS cells from iPS medium to medium consisting of DMEM-low glucose (Biological Industries), $2 \mathrm{mM}$ L-Glutamine, $1 \%$ penicillin/streptomycin, and $10 \%$ FBS (Gibco) as describe previously ${ }^{16}$. The iPSCs began to lose the typical iPSC morphology and acquired a spindle-shaped morphology at the border of the colonies in a few days (Fig. 2a and $2 \mathrm{~b}$ ). After two weeks differentiation, the differentiated iPSCs were passaged with trypsin/EDTA and resulted in a complete morphological change of the cells to a fibroblastic shape after passaging three times (Fig. 2c).

Characterization of hiPS-MSCs. To investigate whether the fibroblastic-like cells differentiated from iPSCs were MSCs, these cells (three passages: 3,5 , and 7) were analyzed by flow cytometry for human mesenchymal makers $\left(\mathrm{CD} 0^{+}, \mathrm{CD} 3^{+}, \mathrm{CD} 105^{+}, \mathrm{CD} 34^{-}\right.$, and $\mathrm{CD}_{45}$ ). The iPS-derived cells were indeed positive for CD90, CD73, and CD105 and negative for CD34, and CD45 in all three passages tested (Fig. $2 \mathbf{d}-\mathbf{2 h}$, results from passage 7 ). These hiPSMSCs were actively proliferating and were further characterized as karyotypically normal after splitting in vitro for 17 passages (Fig. 2i). To test whether these cells loss the pluripotency of iPSCs, we measured the expression of Oct3/4, NANOG, and TRA-1-81 in two different passages ( 8 and 12) of iPS-MSCs by flow cytometry analysis. The hiPS-MSCs were negative for OCT3/4 and TRA-1-81, however positive for NANOG (Fig. 3). We furthermore tested the hiPS-MSC-like cells for MSC pluripotency by investigating their abilities of osteogenesis, adipogenesis, and chondrogenesis. For osteogenesis, hiPS-MSCs (passage 4) were cultured in osteogenic medium and were positive for alkaline phosphatase (ALP) from day 7 (Supplementary Fig. 2b) but not control medium (Supplementary Fig. 2a) and began to mineralize the extracellular matrix (ECM) as determined by Alizarin red staining on day 14 (Fig. 4a). For adipogenesis, Oil Red $\mathrm{O}$ staining demonstrated small lipid droplets in the cytoplasm of differentiated hiPS-MSCs (Fig. 4b). After 3 weeks of culture in chondrogenic medium, pellet cultures of hiPSMSCs demonstrated red-purple proteoglycan-rich extracellular matrix by toluidine blue staining (Fig. 4c), suggesting that the iPSderived cells hold MSCs properties and pluripotency.

The hiPS-MSCs formed calcified structures in scaffolds in vitro. We further explored the possibility of using the hiPS-MSCs for osteogenesis in 3D scaffolds. We firstly functionalized the PCL scaffolds with hyaluronan, which could accelerate tissue repairing by promoting the migration and differentiation of mesenchymal cells $^{17}$, and incorporated the inorganic component $\beta$-TCP to improve the osteoconductivity and enhance bone tissue formation $^{18}$. After fabrication of the PHT scaffolds, Hyaluronan/TCP matrix homogenously covered and connected the PCL scaffold fibers as seen by SEM scanning (Fig. 5a) .

Firstly, we investigated the viability of cells cultured on PHT scaffolds by confocal microscopy with CellTracker green and Hoechst staining. One day after adding the hiPS-MSCs (passage 4) to the PHT scaffolds, all cells were attaching well to the fibers (Fig. 5b). Moreover, after culturing in proliferation medium for one week, the hiPS-MSCs attached to and proliferated along the fibers of scaffolds (Fig. 5c). This indicates that the PHT scaffolds are suitable for hiPS-MSCs growth given that it facilitates hiPS-MSCs initial attachment onto the surface, spreading and subsequent proliferation.

We then investigated the osteogenic capacity of the iPS-MSCs in PHT or PCL scaffolds by measuring the ALP activity and calcium deposition during an osteoinduction period (21 days). Relative ALP activity was calculated by normalizing to DNA contents (Fig. 5d). As seen in Fig. 5d, hiPS-MSCs-seeded scaffolds cultured in osteogenic medium significantly increased ALP activity throughout the osteoinduction period, irrespective of scaffolds. Notably, The ALP activity also increased in hiPS-MSCs-seeded scaffolds cultured in proliferation medium, suggesting that the PCL or PHT scaffolds are capable of inducing ALP expression in MSCs. However, higher ALP activity was observed in hiPS-MSC-seeded PHT or PCL scaffolds cultured in osteogenic medium than in proliferation medium from day 7 onwards (Fig. 5d). ALP activity of the PHT scaffolds was significantly higher than that of PCL in osteogenic medium on day 21 

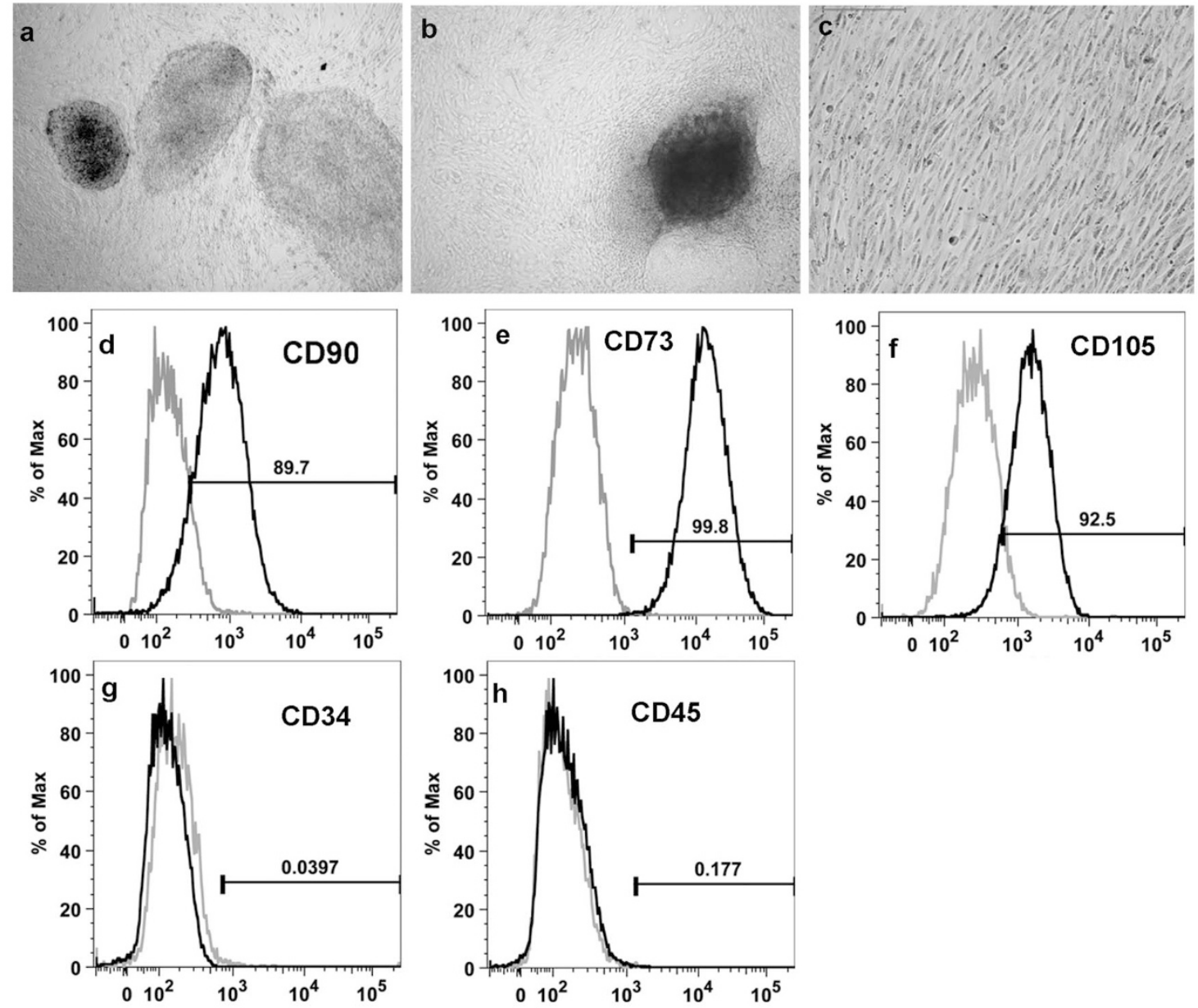

i

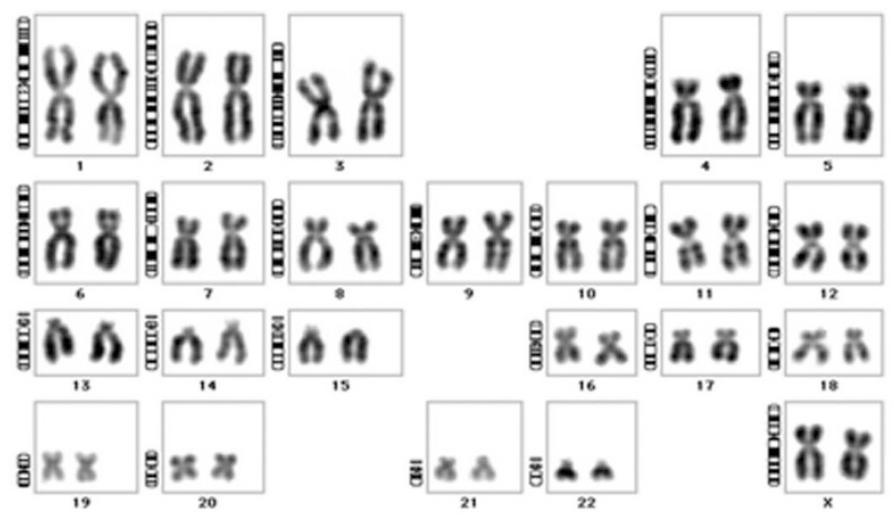

Figure $2 \mid$ Generation and characterization of iPS-derived mesenchymal stem cells. Phase-contrast image of long-term cultured iPSC clone before differentiation (a) intermediate phase of differentiating the iPS cells into MSCs (b) and complete differentiated fibroblast like cells (passage 3) (c). $(\mathrm{d}-\mathrm{h})$ Flow cytometric analysis of mesenchymal markers (one representative of three individual passages, figure shown were from passage 7 hiPS-MSCs). Black histograms: anti-CD90 (d) anti-CD73 (e) and anti-CD105 (f) and negative markers: anti-CD34 (g) and anti-CD45 (h). Gray histograms: corresponding isotype controls $(\mathrm{d}-\mathrm{h})$. (i) karyotype of passage 17 iPS-MSCs.

(Fig. 5d). Calcium contents were measured to assess the extent of matrix mineralization on the scaffolds. Total calcium content was significantly $(\mathrm{p}<0.05)$ higher on days 14 and 21 scaffolds in osteogenic medium as compared to that in proliferation medium (Fig. 5e). Again, we observed the hiPS-MSCs-seeded PHT scaffolds had higher calcium deposition than the corresponding PCL scaffolds $(\mathrm{p}<0.05)$. We also measured the expression of osteogenesis-related genes following the induction of osteogenesis. Increase expression of osteoblast markers, the transcription factor Runx2, alkaline phosphatase $(A L P)$, extracellular matrix and structural proteins coding gene Colla1, and the bone-specific protein-osteocalcin coding gene $(O C)$ were observed in hiPS-MSC-seeded PHT or PCL scaffolds cultured in osteogenic medium than in proliferation medium (Fig. 6). Higher induction of ALP expression was observed in PHT than in PCL scaffolds, which is consistent with the results of the ALP activity assay. 
Passage 8
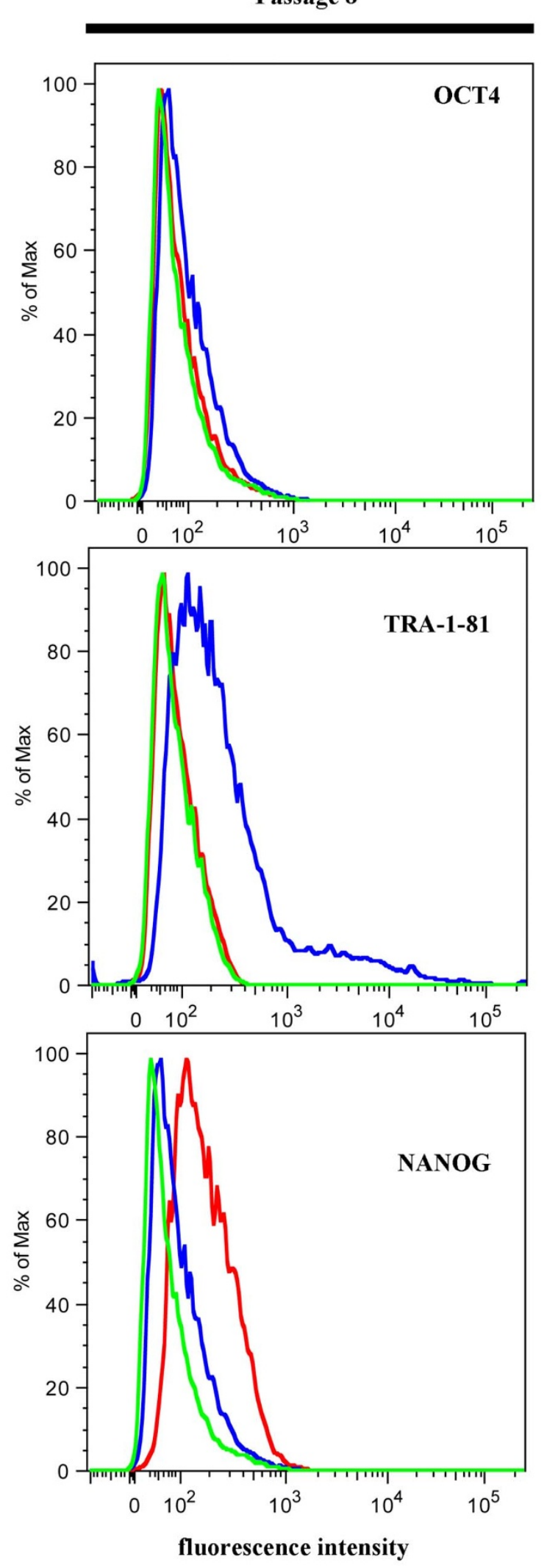

Passage 12
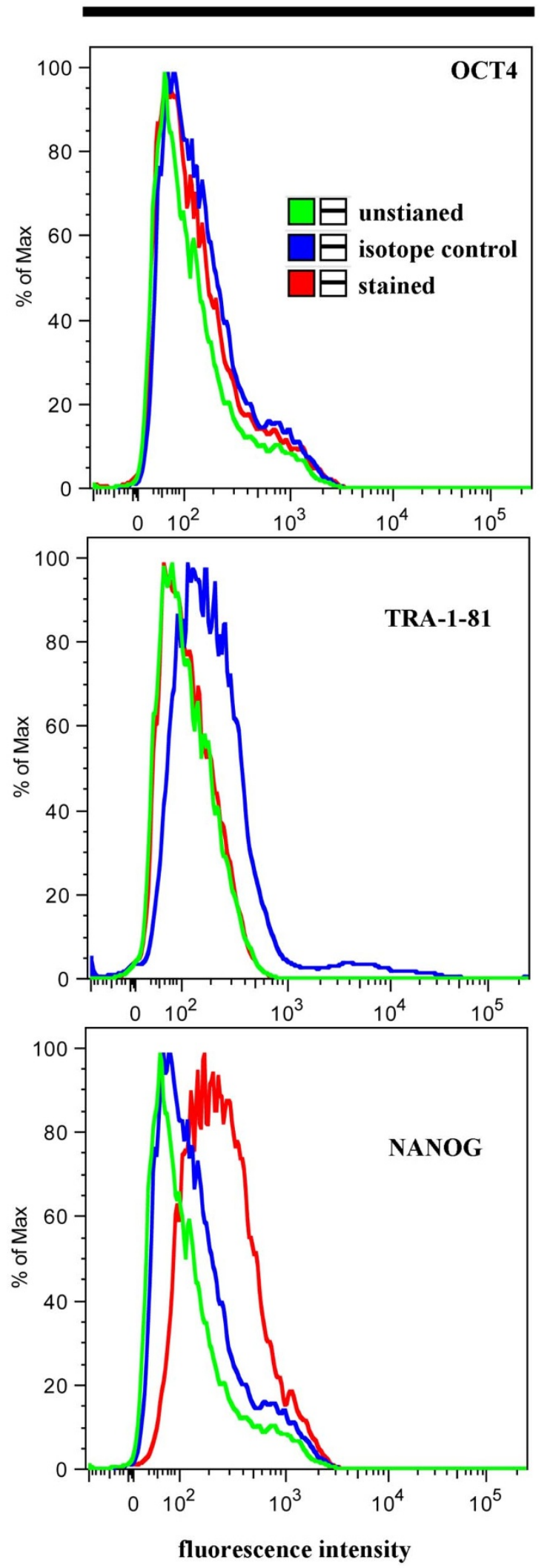

Figure 3 Analysis of pluripotent markers in the hiPS-MSCs. Flow cytometric analysis of pluripotent markers: OCT3/4, NANOG, and TRA-1-81. Green histogram: unstained cell control; Blue histogram: isotope control; Red histogram: stained with markers against OCT3/4, NANOG, and TRA-1-81 respectively. 

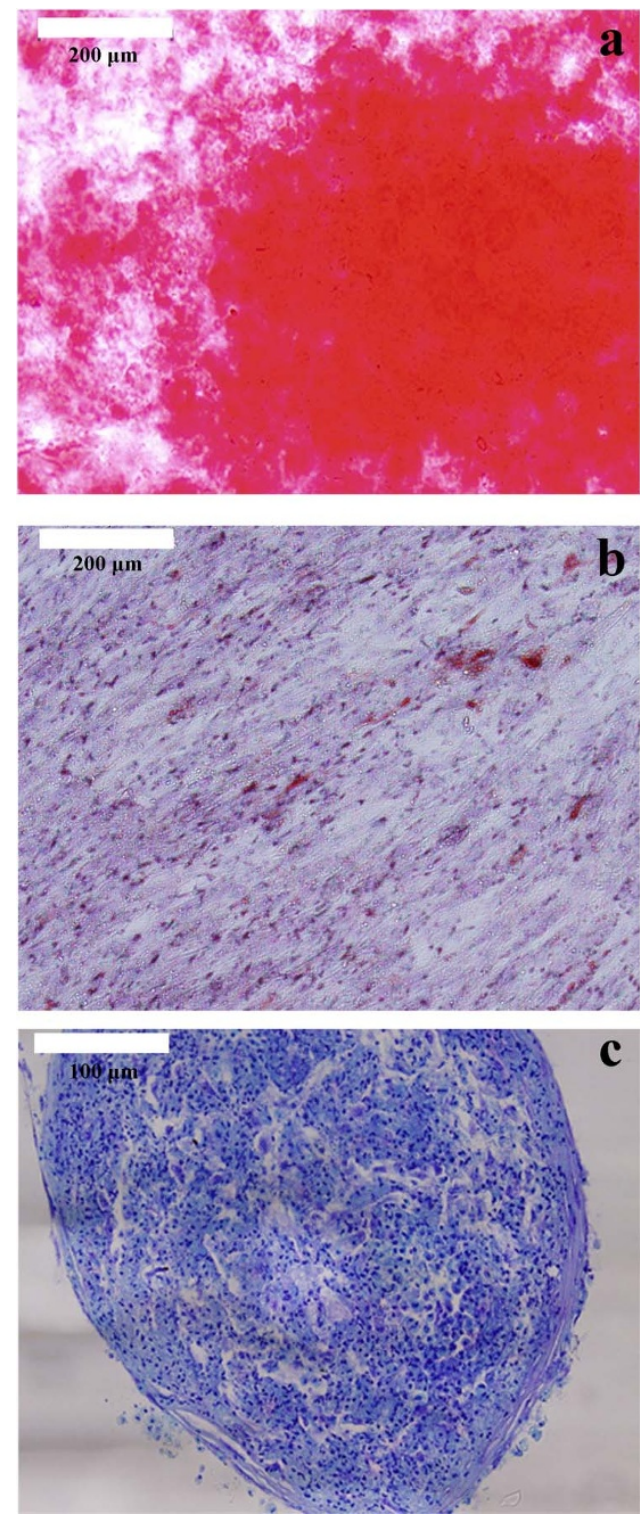

Figure 4 | Tri-lineages differentiation of the hiPS-MSCs. (a) Alizarin red staining of osteogenic mineralization (day 21). (b) Oil Red O staining of small lipid droplets (day 21). (c) Toluidine blue staining of cartilage positive cells (day 21).

We furthermore characterized the formation of ECM at day 21 by SEM. Scaffolds (PCL or PHT) seeded with iPS-MSCs and cultured in basal proliferation medium have a clear cellular network with associated ECM attached onto the rods and spanning across the pores of the scaffolds (Fig. 7a and 7b). ECM formation was observed in the PCL or PHT scaffolds cultured osteogenic medium (Fig. 7c and 7d) or in control medium (Fig. 7a and 7b), respectively. The PCL scaffold is a calcium-free scaffold (Fig. 7a). De novo calcium mineralization was detected on PCL scaffold co-cultivated with iPS-MSCs in osteogenic medium by energy dispersive X-ray spectrometer/EDX (Fig. 7c). The PHT scaffold contains calcium owing to the TCP. Thus, calcium was detected both in proliferation and osteogenic medium (Fig. $7 \mathrm{~b}$ and $7 \mathrm{~d}$ ).

iPS-MSCs form ectopic bone in scaffolds in vivo. Finally, we evaluated whether the iPS-MSCs seeded scaffolds could promote ectopic bone formation in vivo. We subcutaneously implanted one PCL or PHT scaffold with and without preseeded hiPS-MSCs (passage 5) into each side of a nude mouse (Fig. 8a). Four mice were used for each type of scaffolds. Twelve weeks after implantation, the scaffolds were well integrated into the surrounding host tissues, and most importantly, no teratoma was observed. Formation of fibrosis was observed in all scaffolds implanted by trichrome staining, with an indication of high fibrosis in cell-seeded scaffolds than control scaffolds (Fig. 8b-8e). Mineralization was only detected in scaffolds (PCL and PHT) seeded with hiPS-MSCs (Fig. 8d and 8e). We further used micro-CT to quantify the ectopic bone formation. Newly formed bones and typically rod-like trabeculae were observed in all four groups (Fig. 9, left panel). Besides, the ectopic bone from the PHT-iPS-MSCs group was more uniform than other groups (Fig. 9, right panel). We then quantified the microarchitectures among these four groups (Supplementary Table 2). However, due to the limited number of animals used, the only significant differences found were in the case of trabecular separation and trabecular number between the iPS-MSC seeded PCL scaffolds and the control PCL scaffolds.

\section{Discussion}

Over the pass few years, remarkable progress has been made in the generation of clinically compliant and safer human iPSCs by means of virus- and/or vector-free methods. The iPSCs we generated by mRNA reprogramming closely recapitulated the characteristics of human ESCs and are comparable to the iPSCs generated by the same method from other groups ${ }^{7,8}$. We in this study further showed that these iPSCs are an attractive stem cell source for deriving mesenchymal stem cells towards orthopedic applications.

Embryoid body (EB) formation is one of the most broadly used methods for inducing embryonic stem cells (ESCs) or iPSCs towards mesenchymal differentiation. However, the initial EB-mediated differentiation is spontaneous and thus composing all kinds of somatic cells along with the MSCs. Enrichment of MSCs by means of cultivation in MSCs-inducing medium followed by FACS is commonly required after EB differentiation ${ }^{10,12,19,20}$. Embryoid bodyindependent mesenchymal differentiation methods have been developed and optimized during the last few years to facilitate the induction of MSCs from human ESCs/iPSCs. Barberi et al. in 2005 described a simple method of deriving functional multipotent mesenchymal precursors from hESCs by culturing the hESCs on OP9 feeders in the presence of $20 \%$ heat-inactivated FBS in alpha MEM medium followed by CD73-based FACS ${ }^{21}$. The efficiency of CD73 positive cells achieved by their study was relatively low, an average of 5\%. Lim's group applied a modified method by culturing single hESCs on gelatin-coated plates in a conditional that are suitable for MSCs growth, which has greatly improved the efficiency of deriving functional $\mathrm{MSCs}^{22,23}$. Repeated passaging by trypsinization has been showed to enriched MSCs population ${ }^{16,22}$. Other modifications, such as coating tissue culture plate surface with fibrillar collagen $^{24}$ or supplemental small molecule inhibitor ${ }^{25}$, have been showed to facilitate MSCs differentiation from hESCs/hiPSCs as well. The hiPS-MSC-derivation method we used in this study is similar to Yen et al. 's, which was used to derive hES-MSCs ${ }^{16}$.

The hiPS-MSCs we generated approached almost $100 \%$ positive for CD73 (from passage 3), recapitulated mesenchymal stem cell markers and were capable of differentiating into mesenchymal lineages (osteoblasts, adipocytes, and chondrocytes), consistent with report from Yen et al. ${ }^{16}$. Most importantly, the iPS-MSCs are compatible with and functional for osteogenesis in 3D scaffolds both in vitro and in vivo, suggesting that these cells can help evaluating and improving the development of new nano-featured scaffolds in the future. However, mechanisms of why functional MSCs, or mesenchymal progenitor cells named by Yen et al. in their study ${ }^{16}$, can efficiently derived from iPSCs by serial culturing and trypsinization needs further investigations. One possibility might be associated with the serum that we used. However, since we used a different lot of serum from Yen et al. (also from different suppliers), we speculate 

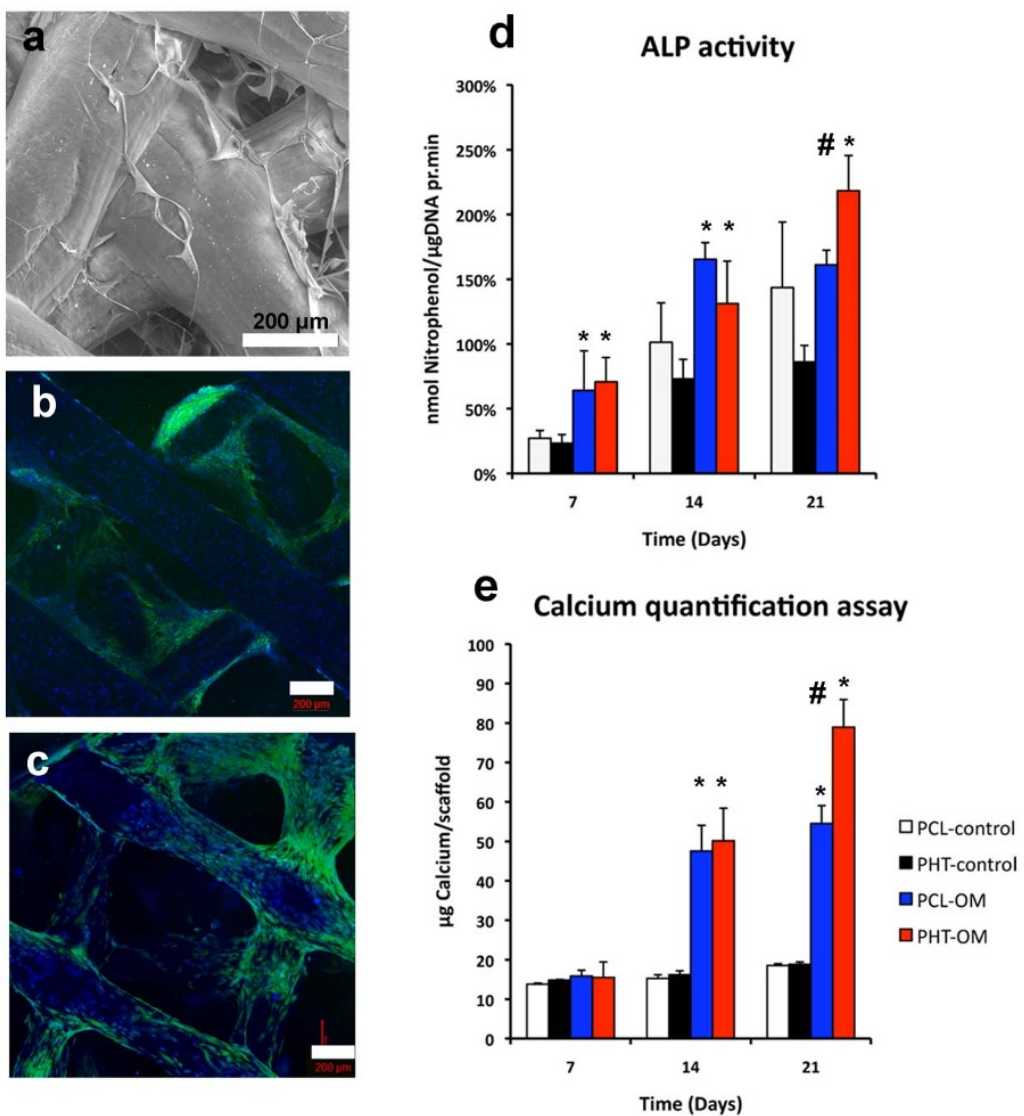

Figure $5 \mid$ Osteogenesis with iPS-MSCs in 3D scaffolds in vitro. (a) SEM image of PHT scaffold shows hyaluronan/TCP matrix covers and connects the PCL scaffold fibers. (b, c) Confocal micrographs of cell viability with CellTracker green and Hoechst staining on PHT scaffolds after one day (b) and 7 days culture (c). Green pixels: live cells; red pixels: nuclei. (d-f) Time-course measurement of ALP activity (d), calcium content (e).

Runx2
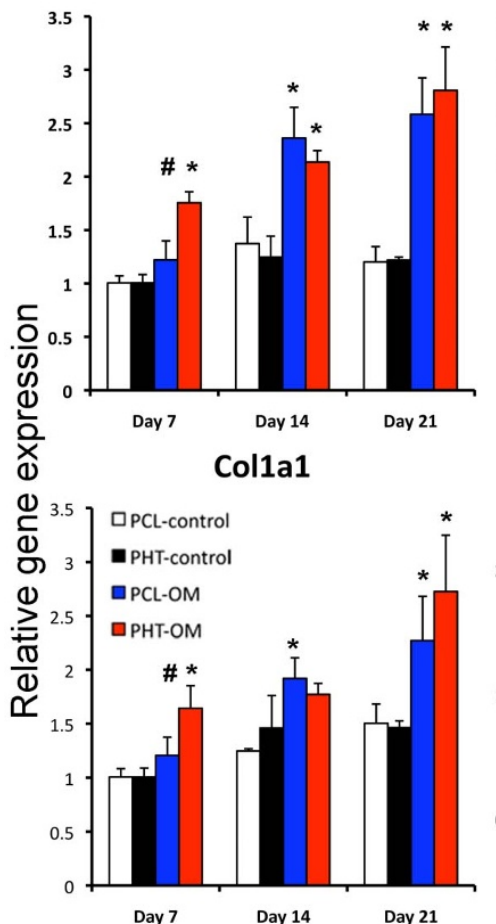

ALP
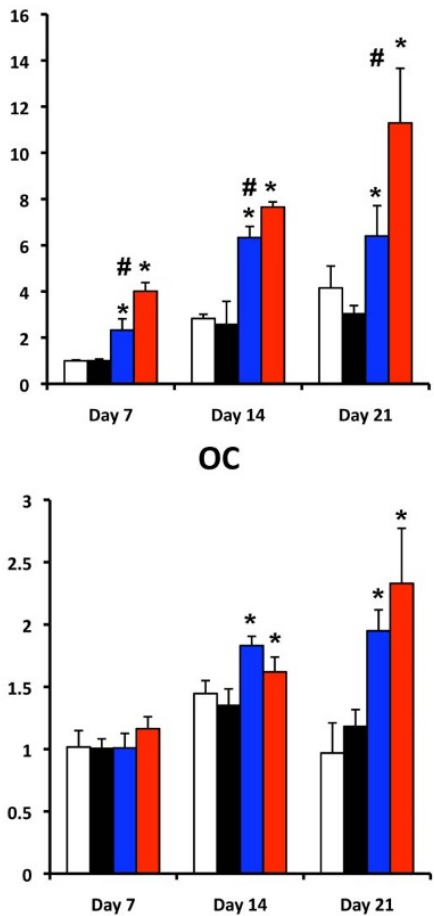

Figure 6 | Analysis of osteoblast markers expression by qPCR. The expression of Runx2, ALP, Col1a1 and OC were determined by qPCR during osteodifferentiation (triplicate experimental repeats). Time-course (7, 14, and 21 days) gene expression was analyzed in hiPS-MSCs-seeded PCL or PHT scaffolds cultivated in proliferation medium (control) or osteogenic medium (OM). *, statistically significant between PCL-OM and PCL-control, PHTOM and PHT-control; \#, statistically significant between PCL-OM and PHT-OM. 


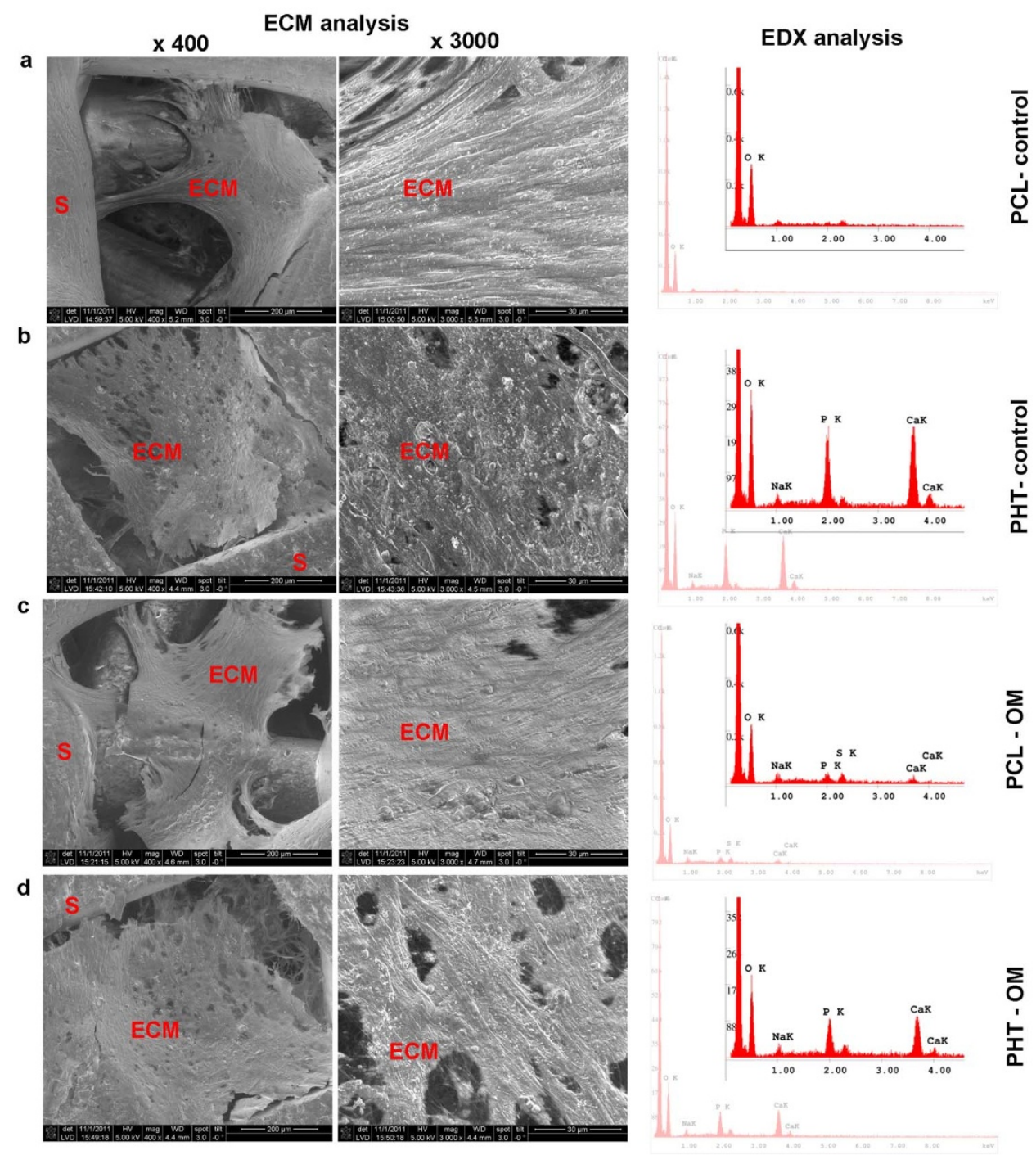

Figure 7 The hiPS-MSCs form calcified structures in 3D scaffolds in vitro. SEM images show extracellular matrix formation in the hiPS-MSCs The expression ofseeded PCL (a) or PHT (b) scaffolds in proliferation medium (control), and the hiPS-MSCs seeded PCL (c) or PHT (d) scaffolds in osteogenic medium $(\mathrm{OM})$ after 21 days culture. Energy dispersive X-ray spectrometer analysis of calcified formation on scaffolds was showed in the right panel. S, scaffold; ECM, extra cellular matrix.

that the serum effect should not be main effector. However, the choice and effect of serum for hiPS-MSCs differentiation needs exploration in a future study. We have also investigated whether this iPS-MSCs differentiation method can be applied to iPSCs generated by other reprogramming method rather than mRNA reprogramming. The same functional hiPS-MSCs were indeed successfully generated from three lines of human iPSCs produced by lentivirusbased reprogramming (data not shown).

Human pluripotent stem cells-derived mesenchymal stem cells have the same in vitro and in vivo characteristics as MSCs derived from adult tissues, such as bone marrow, fat, umbilical cord, and others $^{26,27}$. However, it has been consistently observed that ESCs/ iPSCs-derived MSCs have an advance in growth over adult $\mathrm{MSCs}^{13,28}$. Higher telomerase activity and less senescence were observed in pluripotent stem cells-derived MSCs as compared to adult $\mathrm{MSCs}^{13}$. In this study, we have characterized the loss of pluripotency-as seen with the loss of OCT3/4 and TRA-1-81 - along with the loss of tumorigencity of the iPS-MSCs. However, we still observed the express of Nanog in these iPS-MSCs. Nanog positive adult MSCs or iPS-MSCs have been reported previously ${ }^{25,29}$. Recent study by Han et al. found that expression of Nanog in MSCs was crucial for maintaining MSC proliferation capacity and myogenic differentiation potential ${ }^{30}$, suggesting the expression of Nanog in the hiPS-MSCs derived by our method preserves the proliferative and mesenchymal potential of MSCs to some extent.

Metal implants are widely used in orthopedic surgery, but they are not bioresorbed and have risk of damage to surrounding bone due to stress shielding ${ }^{31}$. Biodegradable materials have gained increased interest as scaffold material because they can be fabricated for a wide range of biomedical applications with a high processability, controlled degradation, adjustable mechanical properties, and possibility for modification ${ }^{32}$. Although polycaprolactone (PCL) scaffold is a popular material since its degradation occurs naturally in the body's metabolism and the degradation compounds do not damage surrounding tissue ${ }^{33}$, PCL is not biologically active it does not stimulate stem cell bone formation. In this study, we also found that supplement of hyaluronan and $\beta$-TCP to the PCL scaffolds (named PHT scaffolds) could enhance the osteogenic capacity of iPS-MSCs, including increase ALP activity, calcium mineralization and ECM, as well as more uniform ectopic bone formation in vivo. More preclinical (in large animals such as pigs) or even clinical trails of the PHT scaffolds may allow this new scaffold to server as new biomaterial for orthopedic applications. The combination of iPS-MSCs and scaffolds holds great potential for patient-specific orthopedic 

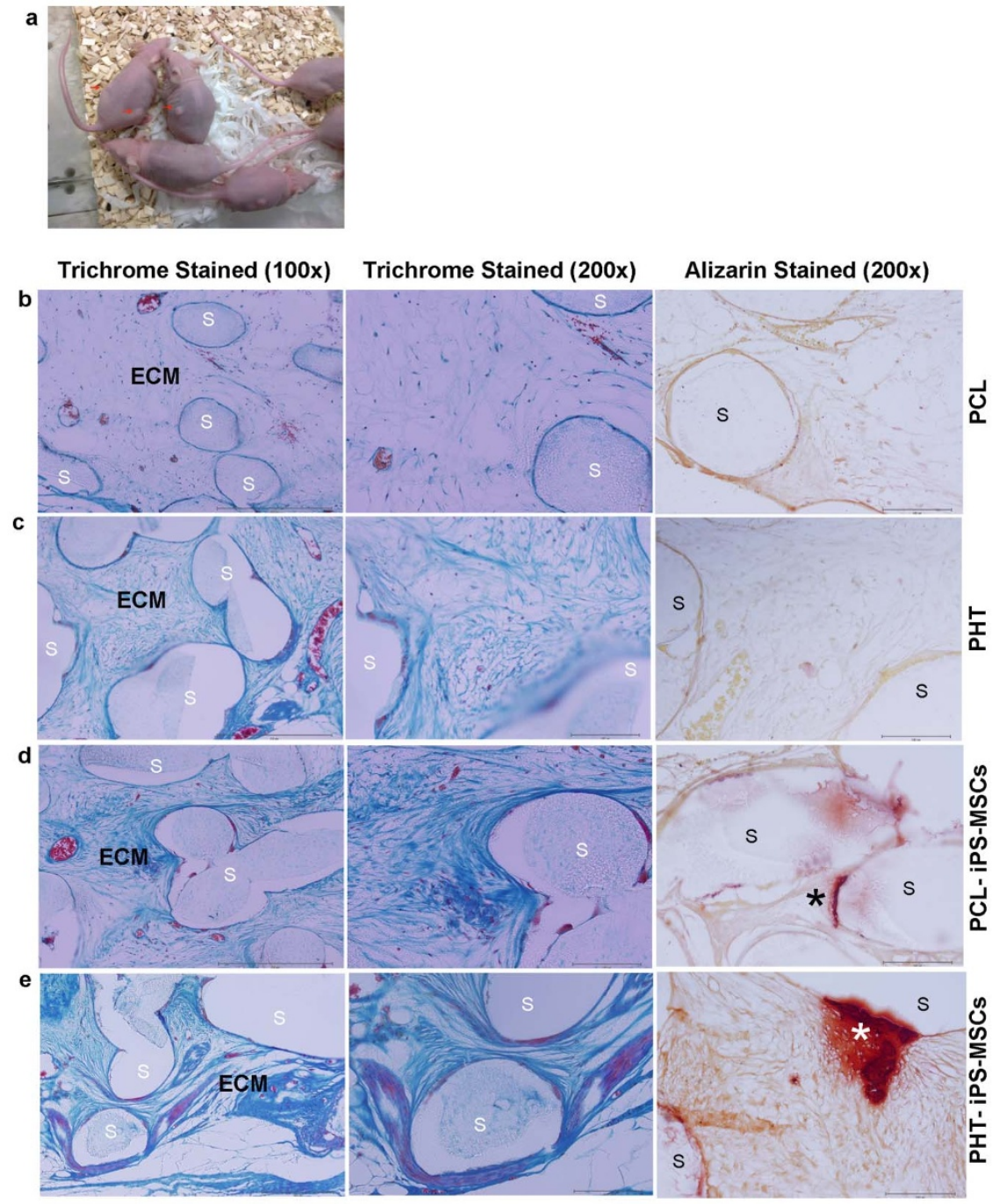

Figure 8 Osteogenesis with hiPS-MSCs in 3D scaffolds in vivo. Picture of nude mice with subcutaneous implantation of scaffolds (a). Trichrome and Alizarin Red staining of cell-free PCL (b) cell-free PHT (c) iPS-MSCs seeded PCL (d) and iPS-MSCs seeded PHT scaffolds (e) after implantation into nude mice for 12 weeks. S, scaffolds; Positive Alizarin Red staining in (d) and (e) was pointed with stars.

therapy, as it was showed that differentiated cells from syngenetic iPSCs could bypass the host immune response ${ }^{34}$. However, this application should requires much more preclinical evaluation and testing, including safety assessment and safer approach as it is in other aspects ${ }^{28}$. Apart from that, our data and many other studies suggest that the iPSCs are a great source of stem cells towards patientspecific mesenchymal stem cell therapy.

\section{Methods}

Reprogramming of human fibroblasts (IMR90). Human primary fibroblasts IMR90 were obtained from ATCC (catalog CCL-186). Reprogramming experiments were performed in 6-well tissue culture plates coated with GlobalStem human feeders at a cell density of $2.50 \times 10^{5}$ per well. The IMR90 cells were plated $(100 \mathrm{~K}$ per well $)$ in Pluriton medium (Stemgent) supplemented with $200 \mathrm{ng} / \mathrm{ml} \mathrm{B18R}$ (interferon inhibitor from eBioscience) throughout the modified mRNA reprogramming cocktail transfection. An RNA dose of $1.2 \mu \mathrm{g}$ was delivered to each well using RNAiMAX (Invitrogen) 4 hours prior to the daily medium change. RNAiMAX transfection cocktails were assembled by diluting $100 \mathrm{ng} / \mathrm{uL}$ RNA $5 \mathrm{X}$ and $5 \mathrm{uL}$ of RNAiMAX per microgram of RNA 10X separately in DPBS without calcium or magnesium. Clones of iPS cells were selected and transferred to fresh human feeder coated plates after 18-20 days of modified mRNA transfection.

Mesenchymal differentiation of iPSCs without EB formation. For the derivation of iPSC-MSCs, three days after splitting, iPS medium was replaced with MSC medium, which consisted of DMEM-low glucose (Biological Industries), $2 \mathrm{mM}$ L-Glutamine, $1 \%$ penicillin/streptomycin, and 10\% FBS (Gibco). The MSC medium was changed every 2 days. After 14 days culture, the cells were trypsinized $(0.25 \%$ trypsin $/ 1 \mathrm{mM}$ EDTA, Difco-Sigma) and expanded in MSC medium on $0.1 \%$ gelatin-coated dishe (Becton Dickinson). When confluent (3-5 days), cells were harvested with $0.025 \%$ trypsin-EDTA and then regularly passaged at a $1: 3$ ratio. Usually, after the third trypsinization, a morphologically homogeneous population of fibroblast-like cells became evident and was used in the analysis of MSC phenotypic characteristics and differentiation potential.

Surface Antigen Analysis of iPS-MSCs. Cell surface antigens of human iPS-MSCs were analyzed by flow cytometry. Cells (passage 3,5, and 7) were harvested by trypsinization from T150 flask followed by two washes with PBS (with 2\% FBS). A total of $5 \times 10^{5}$ cells were stained in a final volume of $100 \mu \mathrm{l}$ PBS/2\% FBS for 30 minutes at room temperature in the dark. The following murine-anti-human antibodies was used at the manufacturer's recommended concentration: anti-CD45 BD Horizon V450, anti-CD90 FITC, anti-CD105 PerCP-Cy5.5, anti-CD73 PE, and anti-CD34 APC (all from BD Biosciences, San Jose, CA). As controls, fluorochrome and concentration matched isotopes were used (all IgG1, BD Biosciences). Following two washes in PBS $/ 2 \% \mathrm{FBS}$, cells were resuspended in $1 \%$ formaldehyde in PBS while vortex mixing. Cells were analyzed using a FACSAria ${ }^{\mathrm{TM}}$ III (BD Biosciences) equipped with a $375 \mathrm{~nm}, 488 \mathrm{~nm}, 561 \mathrm{~nm}$, and $633 \mathrm{~nm}$ laser. For each sample 30,000 cells were collected and subsequently analyzed using FlowJo software (v. 9.3.3, TreeStar Inc., Ashland, OR).

In vitro multipotent differentiation of iPS-MSCs. Multipotency of iPS-MSCs was tested for differentiation along the osteogenic, chondrogenic, and adipogenic lineages. The medium was replaced every 3-4 days for 21 days. The cells cultivated in basal medium served as control. To induce osteogenic differentiation, cells were cultured in osteogenic differentiation medium consisting of DMEM-high glucose 
3. Takahashi, K. et al. Induction of pluripotent stem cells from adult human fibroblasts by defined factors. Cell 131, 861-872 (2007).

4. Thomson, J. A. et al. Embryonic stem cell lines derived from human blastocysts. Science 282, 1145-1147 (1998).

5. Bellin, M., Marchetto, M. C., Gage, F. H. \& Mummery, C. L. Induced pluripotent stem cells: the new patient? Nat Rev Mol Cell Biol 13, 713-726 (2012).

6. Zhou, H. et al. Generation of induced pluripotent stem cells using recombinant proteins. Cell Stem Cell 4, 381-384 (2009).

7. Warren, L. et al. Highly efficient reprogramming to pluripotency and directed differentiation of human cells with synthetic modified mRNA. Cell Stem Cell 7, 618-630 (2010).

8. Yakubov, E., Rechavi, G., Rozenblatt, S. \& Givol, D. Reprogramming of human fibroblasts to pluripotent stem cells using mRNA of four transcription factors. Biochem Biophys Res Commun 394, 189-193 (2010).

9. Stadtfeld, M., Nagaya, M., Utikal, J., Weir, G. \& Hochedlinger, K. Induced pluripotent stem cells generated without viral integration. Science 322, 945-949 (2008).

10. Teramura, T. et al. Induction of mesenchymal progenitor cells with chondrogenic property from mouse-induced pluripotent stem cells. Cell Reprogram 12, 249-261 (2010).

11. Villa-Diaz, L. G. et al. Derivation of mesenchymal stem cells from human induced pluripotent stem cells cultured on synthetic substrates. Stem Cells 30, 1174-1181 (2012).

12. Bilousova, G. et al. Osteoblasts derived from induced pluripotent stem cells form calcified structures in scaffolds both in vitro and in vivo. Stem Cells 29, 206-216 (2011).

13. Lian, Q. et al. Functional mesenchymal stem cells derived from human induced pluripotent stem cells attenuate limb ischemia in mice. Circulation 121, 1113-1123 (2010).

14. Hwang, N. S. et al. In vivo commitment and functional tissue regeneration using human embryonic stem cell-derived mesenchymal cells. Proc Natl Acad Sci U S A 105, 20641-20646 (2008)

Figure 9 | The hiPS-MSCs form ectopic bone in 3D scaffolds in vivo. Three-dimensional reconstruction of micro-CT images for the 4 groups are shown in left panel, thin layers of 5 slices (50 micrometers) are displayed in middle and right panels for illustration purpose. The newly formed bones were distributed nicely in 3D space within scaffolds and trabeculae were typically rod-like.

(Invitrogen) supplemented with 10\% FBS (Gibco), $290 \mathrm{nM}$ ascorbic acid-2phosphate (Merck), $5 \mathrm{mM} \beta$-glycerophosphate (Sigma), and $100 \mathrm{nM}$ dexamethasone (Sigma) for 3 weeks. Calcium deposition was detected by Alizarin red $\mathrm{S}$ (Sigma) following the manufacturer's instructions. Alkaline phosphatase (ALP) was stained with variamine blue B salt (Fluka) and 1-Naphthyl phosphate sodium salt monohydrate (Merk). Adipogenic differentiation was induced by means of adipogenesis inducing medium containing DMEM-high glucose (Invitrogen), 15\% horse serum (Sigma), and $100 \mathrm{nM}$ dexamethasone (Sigma). Cells were examined for the presence of lipid vacuoles by Oil Red $O$ staining using standard techniques as previously described (18). For chondrogenic differentiation, $2.5 \times 10^{5}$ cells were centrifuged in a $15-\mathrm{mL}$ polypropylene Falcon tube to form a pellet which is cultured in chondrogenic medium, consisting of DMEM-high glucose (Invitrogen), $50 \mathrm{mg} / \mathrm{mL}$ ascorbic acid, $100 \mathrm{nM}$ dexamethasone, $1: 100$ ITS premix (BD Biosciences, San Jose, CA), $40 \mu \mathrm{g} / \mathrm{ml}$ L-proline (Sigma-Aldrich), $10 \mathrm{ng} / \mathrm{mL}$ human recombinant TGF $\beta 3$ (R\&D systems). Presence of proteoglycan (PG) was documented by means of toluidine blue staining.

Subcutaneous implantation surgical procedure. Seven-week-old NMRI-Nude mice were purchased from Taconic, Ejby Denmark and housed under pathogen free conditions at the Core Animal Facility of Aarhus University. All procedures were performed according to the Animal Care and Use Committee guidelines at the Aarhus University and approved by the Danish Experimental Animal Inspectorate (License no. 2007/561-1407). Mice were anesthetized with inhaled isoflurane 3,75\%. After inducing general anesthesia, two longitudinal skin incisions were made on the dorsal surface of each mouse, and two subcutaneous pockets were created, into which an iPS-MSC-scaffold construct and an acellular control scaffold were inserted. The skin was closed with interrupted 6-0 nylon sutures. Postsurgical pain treatment was performed by adding buprenorphine to the drinking water (Temgesic ${ }^{\circledR}$, Reckitt Benckiser Healthcare, England) at a dose corresponding to $0.7-1.4 \mathrm{mg} / \mathrm{kg}$ for two days. After 12 weeks, animals were euthanized and the implants were fixed in $10 \%$ neutral buffered formalin for histological and micro-CT analysis.

Other methods used in this study were provided in supplementary file. Primers used for Q-PCR were listed in Supplementary Table 1.

1. Horwitz, E. M. et al. Transplantability and therapeutic effects of bone marrowderived mesenchymal cells in children with osteogenesis imperfecta. Nat Med 5, 309-313 (1999).

2. Minguell, J. J., Erices, A. \& Conget, P. Mesenchymal stem cells. Exp Biol Med (Maywood) 226, 507-520 (2001).

15. Yen, M. L. et al. Efficient Derivation \& Concise Gene Expression Profiling of Human Embryonic Stem Cell-Derived Mesenchymal Progenitors (EMPs). Cell Transplant 20, 1529-1545 (2011).

16. Yen, M. L. et al. Efficient Derivation \& Concise Gene Expression Profiling of Human Embryonic Stem Cell-Derived Mesenchymal Progenitors (EMPs). Cell Transplant, 1529-1545 (2011).

17. Laurent, T. C., Laurent, U. B. \& Fraser, J. R. The structure and function of hyaluronan: An overview. Immunol Cell Biol 74, A1-7 (1996).

18. LeGeros, R. Z. \& Craig, R. G. Strategies to affect bone remodeling: osteointegration. J Bone Miner Res 8 Suppl 2, S583-596 (1993).

19. Wei, H. et al. One-step derivation of cardiomyocytes and mesenchymal stem cells from human pluripotent stem cells. Stem Cell Res 9, 87-100 (2012)

20. Brown, S. E., Tong, W. \& Krebsbach, P. H. The derivation of mesenchymal stem cells from human embryonic stem cells. Cells, tissues, organs 189, 256-260 (2009).

21. Barberi, T., Willis, L. M., Socci, N. D. \& Studer, L. Derivation of multipotent mesenchymal precursors from human embryonic stem cells. PLoS medicine 2, e161 (2005).

22. Lai, R. C., Choo, A. \& Lim, S. K. Derivation and characterization of human ESC-derived mesenchymal stem cells. Methods Mol Biol 698, 141-150 (2011).

23. Choo, A. \& Lim, S. K. Derivation of mesenchymal stem cells from human embryonic stem cells. Methods Mol Biol 690, 175-182 (2011).

24. Liu, Y., Goldberg, A. J., Dennis, J. E., Gronowicz, G. A. \& Kuhn, L. T. One-step derivation of mesenchymal stem cell (MSC)-like cells from human pluripotent stem cells on a fibrillar collagen coating. PLoS One 7, e33225 (2012).

25. Chen, Y. S. et al. Small molecule mesengenic induction of human induced pluripotent stem cells to generate mesenchymal stem/stromal cells. Stem cells translational medicine 1, 83-95 (2012).

26. Gruenloh, W. et al. Characterization and in vivo testing of mesenchymal stem cells derived from human embryonic stem cells. Tissue Eng Part A 17, 1517-1525 (2011).

27. Jung, Y., Bauer, G. \& Nolta, J. A. Concise review: Induced pluripotent stem cellderived mesenchymal stem cells: progress toward safe clinical products. Stem Cells 30, 42-47 (2012).

28. Tsuji, O. et al. Therapeutic potential of appropriately evaluated safe-induced pluripotent stem cells for spinal cord injury. Proc Natl Acad Sci U S A 107, 12704-12709 (2010).

29. Pierantozzi, E. et al. Pluripotency regulators in human mesenchymal stem cells: expression of NANOG but not of OCT-4 and SOX-2. Stem Cells Dev 20, 915-923 (2011).

30. Han, J. et al. Nanog reverses the effects of organismal aging on mesenchymal stem cell proliferation and myogenic differentiation potential. Stem Cells $\mathbf{3 0}$, 2746-2759 (2012).

31. Bobyn, J. D. et al. Clinical validation of a structural porous tantalum biomaterial for adult reconstruction. J Bone Joint Surg Am 86-A Suppl 2, 123-129 (2004).

32. Peter, S. J., Miller, M. J., Yasko, A. W., Yaszemski, M. J. \& Mikos, A. G. Polymer concepts in tissue engineering. J Biomed Mater Res 43, 422-427 (1998).

33. Sun, H., Mei, L., Song, C., Cui, X. \& Wang, P. The in vivo degradation, absorption and excretion of PCL-based implant. Biomaterials 27, 1735-1740 (2006). 
34. Guha, P., Morgan, J. W., Mostoslavsky, G., Rodrigues, N. P. \& Boyd, A. S. Lack of Immune Response to Differentiated Cells Derived from Syngeneic Induced Pluripotent Stem Cells. Cell Stem Cell 12, 407-412 (2013).

\section{Acknowledgements}

We thank Anette Baatrup and Lisa Feng for their great technical assistance. This project is supported by grants from the Danish Strategic Research Council (Individualized Musculoskeletal Repair Network, Jr. nr. 09-063120), Lundbeck Foundation Nanomedicine Center for Individualized Management of Tissue Damage and Regeneration (LUNA)", NSFC (81000676), and Velux (25906).

\section{Author contributions}

L.Z., Y.L., M.C., G.W. and C.B. conceived the idea. L.Z., Y.L. and M.C. designed the experiments, L.Z., Y.L., M.C., G.W., M.D., C.C.P., R.K., F.D., Y.Z., N.L., Q.M. and D.Q. performed experiments. L.Z., Y.L. and M.C. wrote the manuscript and prepared the figures. Y.L., F.B., L.B., T.G.J., J.K., W.T.P. and C.B. supervised and supported the study. Y.L. coordinated the study. All authors reviewed the manuscript.

\section{Additional information}

Supplementary information accompanies this paper at http://www.nature.com/ scientificreports

Competing financial interests: The authors declare no competing financial interests.

How to cite this article: Zou, L.J. et al. A simple method for deriving functional MSCs and applied for osteogenesis in 3D scaffolds. Sci. Rep. 3, 2243; DOI:10.1038/srep02243 (2013).

(c) (i) $(-)$ This work is licensed under a Creative Commons Attribution-

By NC No NonCommercial-NoDerivs 3.0 Unported license. To view a copy of this license, visit http://creativecommons.org/licenses/by-nc-nd/3.0 